\title{
MindTick: Case Study of a Digital System for Mental Health Clinicians to Monitor and Support Patients Outside Clinics
}

\author{
Rebecca PERRY ${ }^{\mathrm{a}, \mathrm{b}}$ Lydia OAKEY-NEATE ${ }^{\mathrm{a}, \mathrm{b}}$ John FOUYAXIS ${ }^{\mathrm{a}, \mathrm{b}}$ \\ Sue BOYD-BRIERLEY ${ }^{c}$ Megan WILKINSON ${ }^{c}$ Michael BAIGENT $^{c}$ \\ and Niranjan BIDARGADDI ${ }^{\mathrm{a}, \mathrm{b}, 1}$ \\ ${ }^{a}$ Flinders Health \& Medical Research Institute, Flinders University \\ ${ }^{b}$ Flinders Digital Health Research Centre, Flinders University \\ c Southern Adelaide Local Health Network
}

\begin{abstract}
The current COVID-19 pandemic has highlighted the limitations of relying solely on in-person contact for diagnosis, monitoring and treatment of mental health conditions. Mobile health approaches can be used to monitor mental health patients remotely, but they are not properly integrated with existing models of healthcare service delivery. We present findings from a case study of a mobile app enabled cloud-based software program rolled out in a phone based psychological service to enable real-time/temporal monitoring. The program offered patients an app to record measures of symptoms in everyday contexts and provided clinicians with access to an accompanying dashboard to use information from the app to tailor treatments and monitor progress and ultimately facilitate earlier and personalised care decisions. Feedback related to implementation and utility was gathered from clinicians through a focus group conducted two months post-roll-out. Findings identified that the system is valuable and feasible, however implementation issues were identified. These are discussed in order to inform future work in this area to support the delivery of timely and responsive mental health care in the community.
\end{abstract}

Keywords. Mental health, real-time monitoring, implementation

\section{Introduction}

The COVID-19 pandemic has led to social isolation, restricted movement and disruption to traditional health services throughout the community. Although these stressors affect everyone, it is people with existing mental illnesses who are likely to suffer disproportionately due to higher levels of social disadvantage and poorer underlying physical health [1]. Mental health clinicians cannot offer routine clinics or home visits to conduct mental health assessments during pandemic-like situations. With limited monitoring, these deteriorating patients will go undetected and relapse can occur, substantially contributing to mental health care costs through utilisation of emergency services and psychiatric hospitalisations [1]. The need for sensitive monitoring that can be deployed during pandemic-like situations is therefore necessary for both health and economic reasons.

Monitoring is integral to adequately manage mental illnesses. Routine outcome monitoring to measure change and improve clinical practice is a mandated policy in the

1 Corresponding Author, Niranjan Bidargaddi, Flinders Health and Medical Research Institute, Flinders Digital Health Research Centre, Flinders University, Adelaide; Email: Niranjan.bidargaddi@flinders.edu.au 
public mental health services of all states following the National Mental Health Plan agreed between Australian and State Governments in 2002 [2]. In current practice, symptoms and functioning of registered patients are monitored at fixed-time intervals by clinicians using a set of standardised instruments that rely on patient report of past experiences or expert observations in controlled settings [3]. These approaches yield only a periodic "snapshot" rather than a time-varying record of a person's symptoms, behaviour and functioning that is necessary to detect emerging decline early. The ability to administer these forms of assessment is further diminished in the aftermath of extreme health events such as the pandemic, as these assessments are typically conducted in person and at treatment facilities that may no longer be feasible e.g. due to social distancing requirements or other circumstances. Furthermore, it has been demonstrated that self-report instruments, even if validated measures, are subject to recall bias, function overestimation, social desirability effects and state-dependent biases [4]. Expert observations made in a controlled clinic setting are also not reflective of how patients function and experience symptoms in their home during daily life/the real world. Both approaches lack the temporal resolution necessary to detect subtle changes in a patient's status [5]. Consequently, the benefits of outcome monitoring at fixed time points are in doubt, as affirmed by a Cochrane meta-analysis [6].

The pandemic has highlighted mental health care providers have a pressing need for alternative ways to monitor and support patients digitally and remotely [7]. Changes in activity, sleep, mood, social interaction and medication adherence are early warning signs that often precede relapse in people with severe mental illness. These changes can now be identified from evidence-based monitoring technologies [8]. Growing literature in mental health suggests that mobile phones can be used to monitor time-sensitive mental health changes in real-time and remotely [9]. This can be easily achieved through repeated passive and active data collection via a mobile phone application (app). Passive approaches utilise automatically-captured sensor signals in the devices, which are associated with individuals' daily behaviours [10]. For example, the temporal patterns in location and movement sensor measures have been shown to predict changes in mood symptoms and functioning levels [11]. Patients can also actively report symptoms on their mobile phone application in real time, close to experiences, which overcomes recall bias and captures intra-subject variability [4].

The feasibility and validity of mobile device data collection has been demonstrated in individuals with depression [12], bipolar [13] and schizophrenic disorders [14]. Work led by our team shows clinician support for using technology to monitor patient progress and risk in real time, provided it is well integrated into clinical practice [15]. Continuously monitoring a patient's experience of symptoms and behaviour closer in time to real-life events can capture warning signs of suicidal ideation weeks in advance [16], or phase change onset in bipolar disorder [17,18]. Evidence from randomised controlled trials (RCTs) shows the use of real-time monitoring measures to guide management improves patients' symptom outcomes and reduces hospitalisations $[19,20]$.

Despite the evidence for the effectiveness and feasibility of continuous, real-time monitoring of mental health symptoms to inform care, such a model of care is yet to be utilised in traditional health service settings. To address this gap, we have developed an app-based monitoring system (MindTick) specifically designed to make data available to mental health clinicians in a real-world clinical service. 


\section{Description of MindTick}

The MindTick system gathers real time functional and symptomatic measures from patients through apps installed on their mobile phones which can be charted over time on an online dashboard viewable by clinicians. As a cloud-based solution, MindTick is designed to be delivered at scale with the ability to be tailored to support the delivery of services addressing various health conditions. The MindTick system comprises the following components.

\subsection{Monitoring app for patients}

The app extracts passive data from the phone's on-board sensors and applies analytical algorithms to convert these signals into time-stamped measurements of behaviours (e.g., sleep, activity, mobility, social interactions). Through eight validated questions, it actively prompts users at random and weekly intervals to report subjective momentary experiences on symptoms (mood, sleep, alcohol cravings, appetite) and functioning (work, social, family) [21] (Figure 1).
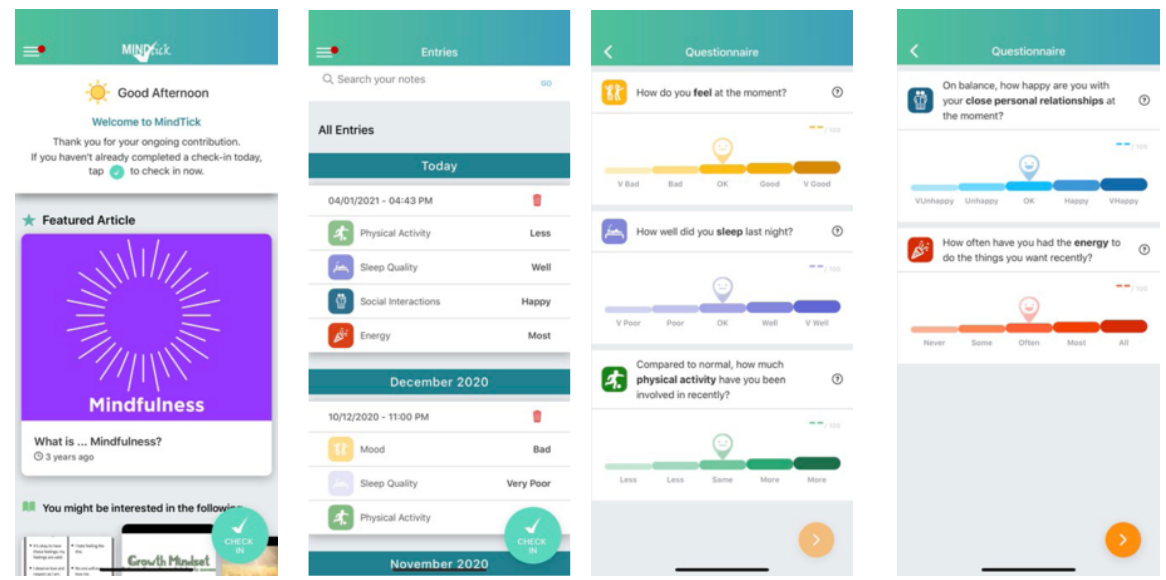

Figure 1. Screen shots of patient app

\subsection{Feedback dashboard for clinicians}

A web-based clinician dashboard accompanies the app and allows both patients and clinicians to access the data and visualise it (Figure 2). It presents each monitored variable online graphs, making interdependencies and time patterns visible. Once registered, clinicians can log in at any time and view their patients' latest dashboards. 

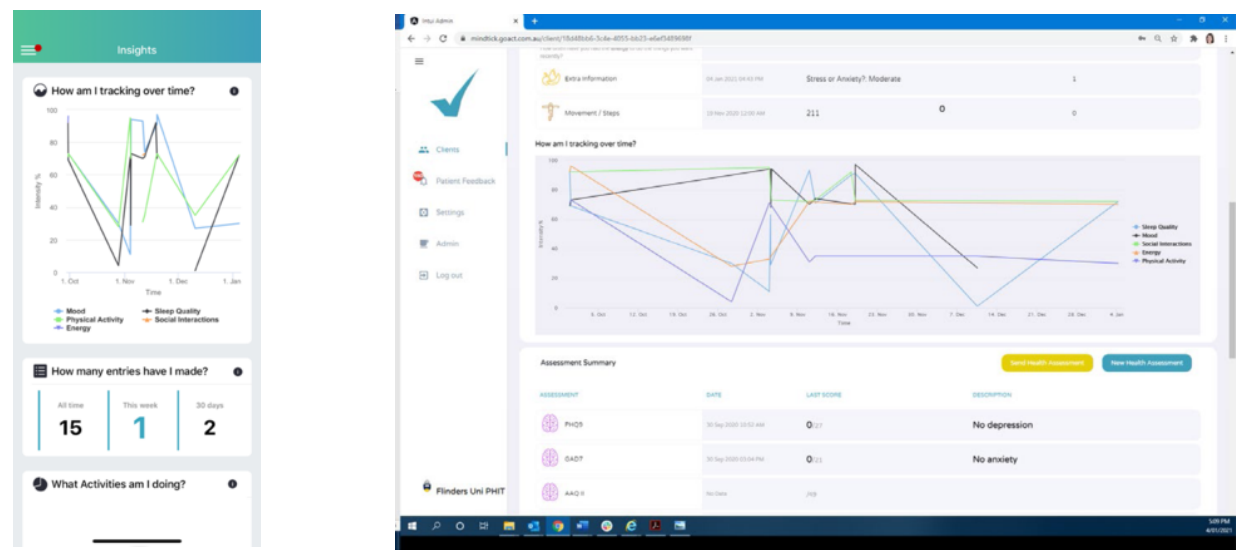

Figure 2. Screen shots of data visualisations in the patient app and clinician dashboard

Integrated security and privacy features in the app and dashboard, already reviewed and approved by two ethics committees in previous research, ensure that sensitive data is securely collected, stored and shared, and complies with the Australian Privacy Act 1988.

In order to understand potential benefits and barriers to adoption of such a system within mental health services within Australia, we present a case study describing the roll-out of MindTick with a real-world phone-psychotherapy clinic and report the learnings gained from a focus group conducted with clinicians who used the system to explore issues associated with implementation processes, utility and acceptability.

\section{Case Study Methodology}

\subsection{Description of the service where MindTick was implemented}

The roll-out site was the Improving Access to Psychological Therapies (IAPT) service based at the Flinders Medical Centre (FMC) in Adelaide, South Australia. The IAPT service offers phone-based 'low intensity' psychological therapies to individuals aged over 18 years who present to FMC ED in psychological distress. Exclusion criteria for IAPT intervention includes those who are actively suicidal, psychotic, taking benzodiazepine over 10mg diazepam (or equivalent) daily, alcohol dependency, heavy illicit drug use, homelessness, under the age of 18 years and under the care of Community Mental Health Team and those already receiving psychological therapy. The IAPT service comprises four therapists who provide a service to around 150 patients per year.

Referred patients have an initial face-to-face assessment and receive weekly telephone sessions and bibliotherapy with guided self-help led by a brief intervention therapist for 6-7 sessions. This evidence-based brief Cognitive Behaviour Theory is considered a pragmatic, efficient, and cost-effective intervention delivery, especially for low intensity mental health issues. 


\subsection{Implementation process}

Prior to commencement of the trial, an information session was held on site with IAPT clinicians to discuss the implementation of MindTick and how the platform could be integrated into daily workflow with minimal clinician burden.

Clinicians were the first point of contact with potential participants and deemed whether they would be suitable to trial MindTick. Participants were required to be accepted for treatment at IAPT for a mental illness as defined in the DSM-5 or ICD-10 and own an android or iOS compatible smartphone. There was no exclusion on gender or ethnicity, however, patients with insufficient ability to understand the study instructions, or with a documented cognitive impairment, substance abuse, or hazardous use of alcohol were excluded. Additionally, patients judged as not able to participate by their treating clinician (based on their assessment that participation would present risks to the patient) were also excluded.

Clinicians were briefed on how to introduce MindTick during the session, and details of suitable and interested patients were passed to a recruiter based at the clinic. The recruiter contacted the patient and guided them through consent and enrolment. Implementation of MindTick was approved by the Southern Adelaide Clinical Human Research Ethics Committee (SAC HREC EC00188): HREC/18/SAC/168.

Prior to each treatment session, IAPT clinicians viewed the dashboard to ask patients more nuanced questions about insights over the period between appointments, and they would record these insights in their clinical information system. The roll-out was undertaken between July and September 2019.

\subsection{Exit interviews with IAPT clinicians}

Following the roll-out of the MindTick system, four clinicians participated in a focus group in December 2019. Focus group sessions were conducted by second and third authors to a) explore how MindTick was used by clinicians and the value and impact it had, b) how MindTick was introduced to patients, c) barriers and enablers of use, and d) open discussion re: recommendations. Sessions were audio-recorded and transcribed verbatim. Transcripts were analysed in NVivo (QSR International Pty Ltd. NVivo (version 10), 2012) by second author using thematic analysis techniques described by Braun and Clarke [22] to identify recurring patterns relevant to understanding clinician's experiences of using the MindTick system. First and last author then defined the final themes to illustrate the key learnings from the focus group discussion.

As indicators of clinical severity of enrolled patients, measurement values of assessments administered at the commencement of routine practice were also recorded (Patient Health Questionnaire scale (PHQ9) [23], General Anxiety Disorder 7-item (GAD-7) [24] and Work and Social Adjustment Scale (WSAS) [25]).

\section{Results}

\subsection{Overview}

Four clinicians were signed up to use the MindTick system in their practice. They introduced the app to 18 patients between July and September 2019. Five patients installed the app (female $\mathrm{n}=4$, age $=26.6(\mathrm{SD}=8.8)$ years $)$. Baseline scores for PHQ- 
9, GAD-7 and WSAS were 18.20 ( $\mathrm{SD}=8.47), 12.40(\mathrm{SD}=6.23), 28.20(\mathrm{SD}=8.90)$, respectively, indicating the presence of depression, anxiety and moderately severe work and social functional impairment. Of a possible 6 sessions, the average attendance rate was $3.40(\mathrm{SD}=2.41)$.

\subsection{Experiences of clinicians}

Four main themes relating to incorporating MindTick into mental health service delivery emerged from the focus group discussion with clinicians. These are presented below along with supporting quotes.

\subsection{Implementation process issues}

a) Barriers

Clinicians reported that patient recruitment was the most difficult aspect of implementation. The majority of patients were extremely overwhelmed or distressed at their first appointment with the mental health service and were reluctant to consider using the MindTick system at that time: "There's a lot going on for people when they're initially referred... people might just say "yes" to get a service, not necessarily to participate in the research." "Offering it right off the bat when people first come in and are really distressed may not be the best time."

Clinicians found it difficult to remember to integrate the MindTick system into practice, especially during the first session with patients, "I found it hard to embed... keep at the forefront of my mind as there is a lot to cover in the initial assessment."

\section{b) Enablers}

Once clients were using the system, mental health clinicians reported very positive experiences regarding implementation processes, "Once they (clients) are signed up ...it is fairly streamlined ... behaviour we can just maintain. That's not anything we would find difficult." "It (the dashboard) put into context what she (client) was telling me, and so did the feedback report."

\subsection{Utility of Data}

Clinicians highlighted several examples where the data collected and presented by the MindTick system was useful (especially visual representation i.e. line graphs); "She (client) was able to see that because she was physically unwell at the time, that her physical symptoms were impacting on sleep and how that was impacting on her anxiety." "I think when they (clients) have something that's a visual...correlation between maybe say sleep and mood... having that visual is a good way for people to actually make most correlations." "One client went through all six sessions and liked the app a lot-used the reports. This was useful because there was a space of a fortnight between sessions...she was feeling good at the session time and many of the stressors had been forgotten. She was able to reflect on that fortnight using the reports as her measures went up and down." "It was making him more aware of his mood and its relationship to sleep and levels of activity...doing what your app is designed to do."

The MindTick system integrated well into the IAPT service, with clinicians reporting that the dashboard data informed the triaging and discharging of patients; 
"We had a client who had disengaged from treatment, but she was still entering on the MindTick app. It showed that her mood was actually declining so that did influence us in what we did in our discharge process to advise the GP that she was possibly in need of support and we asked the GP to book an appointment with her"

Clinicians also found that the reports produced by the MindTick system were easy to interpret and the data helped to inform practice, "It verified what my impression was of the client in terms of her progress and in terms of her response to her stressors. I think that was the most useful aspect of it."

\subsection{Environmental context}

At the time of the trial, the IAPT service was experiencing decreased referrals due to the COVID-19 pandemic; "Don't think it is the time to continue this with the current pandemic, think we could reassess down the track." "At that time there were... quite a lot of DNA (did not attend), people who didn't end up attending the assessment."

In addition, the majority of clients were highly distressed and perhaps not appropriate, "These people present to ED and are referred to IAPT within a week so these are very distressed people with incredibly complex issues". "The majority didn't see that they could actually then manage an app as well as the workbook." "...when there are so many other issues going on e.g. suicidal ideation etc participating in a research project may not be top priority."

\subsection{Recommendations}

The clinicians made a number of suggestions regarding processes and design to improve the adoption of MindTick, "As a value-add, maybe sending a text message as an appointment reminder via the app." "There could be some sort of motivational push to keep them (client) engaged." "...start it at session 3 and then get your data that way, when the initial distress is lower."

\section{Discussion}

We have presented findings from the roll-out of MindTick within a routine public mental health service. The MindTick system gathers real-time functional and symptomatic measures from patients through apps installed on their mobile phones and charts these measures over time on an online dashboard viewed by clinicians. Clinicians working in the service provided feedback on the roll-out experience in a focus group discussion and identified barriers and enablers to implementation that will be useful to inform future work.

Clinicians reported the main barriers to implementation were related to patient recruitment and "embedding" new processes into their practice. The challenges associated with recruiting mental health patients into research trials are well recognised [26] and can be compounded by poor motivation levels typical of illnesses such as depression and schizophrenia [27]. In our case study, patients were recruited from the emergency department of a large metropolitan tertiary hospital and were extremely distressed. Future work should evaluate the suitability of this target group and consider recruiting less complex, less acute patients. 
Challenges around "embedding" MindTick processes into practice relate to adoption as defined in the RE-AIM framework ie. the absolute number, proportion, and representativeness of settings and staff who are willing to offer a program [28]. Strategies to improve adoption include highlighting the program's ability to attract people to use the service and identifying existing resources and practices that would make it easy to deliver the program, including assessing clinicians' level of expertise to do so. It is debatable as to whether the mental health clinicians' current working paradigm allows the incorporation of the detailed patient information provided by the MindTick system into their treatment responses. This is a phenomenon explored by Feijt et al [29] who developed the Levels of Adoption of eMental Health model to provide a structured representation of factors affecting the uptake of eMental health practices by clinicians. Future work should explore this further to ensure both clinicians and patients benefit from eMental health services.

If recruitment barriers were overcome, clinicians reported that the MindTick system was very easy to use. They also reported good clinical utility of the data presented in the clinician dashboard. The data provided context and nuance to patient-reported symptoms and helped to inform practice and discharge processes. Documenting and disseminating these positive experiences to colleagues will also help to enhance adoption [30].

The environmental context aspects affecting the delivery of the trial as reported by clinicians were due to factors intrinsic and extrinsic to the service. Intrinsically, clientele have a tendency to not attend appointments and are also extremely distressed at their initial appointment. Extrinsically, the roll-out occurred during the COVID-19 pandemic at a time when the service was under considerable additional pressure. These factors contributed to the low numbers of participants which must be considered as a limitation of this case study.

The recommendations made by clinicians regarding future delivery of MindTick are very valuable as they have an intimate understanding of clinic processes and their clientele. Similar suggestions were also made following our work developing a digital recruitment strategy in the mental health research setting [31]. These recommendations emphasise that careful consideration must be given to future recruitment processes. The additional features suggested by clinicians e.g. reminders for appointments, homework etc should be considered as they are also likely to improve adoption of the system by clinicians and adherence by patients.

\section{Conclusion}

Mental health services assess patients at fixed intervals and rely very much on patient memory, which is less accurate, prone to biases and not suited to detect early warning signs. Facilitating clinicians to continuously collect and review patients' symptom and functional measurements will enable early identification of relapse indicative symptoms, and hence earlier treatment, which, in turn, may improve level of functioning and quality of life. By addressing implementation barriers identified in this case study, MindTick has the potential to enhance clinicians' capacity to recognise change in symptoms and functioning in near real time and improve care management for people with mental illnesses. 


\section{References}

[1] Australian Institute of Health and Welfare. Mental health services: in brief 2018. Cat. no. HSE 211. AIHW, Canberra. 2018

[2] Department of Health and Ageing. National mental health information priorities, $2^{\text {nd }}$ edition. Commonwealth of Australia: Canberra. 2005.

[3] Pirkis J, Burgess P, Coombs T, Clarke A, Jones-Ellis D, Dickson R. Routine measurement of outcomes in Australia's public sector mental health services. Aust New Zealand Health Policy. 2005 Apr 19; 2(1): 814.

[4] Moore RC, Depp CA, Wetherell JL, Lenze EJ. Ecological momentary assessment versus standard assessment instruments for measuring mindfulness, depressed mood, and anxiety among older adults. J Psychiatr Res. 2016 Apr; 75: 116-123.

[5] Golay P, Basterrechea L, Conus P, Bonsack C. Internal and predictive validity of the French Health of the Nation Outcome Scales: need for future directions. PLoS ONE. 2016 Aug 2; 11(8): e0160360.

[6] Kendrick T, El-Gohary M, Stuart B, Gilbody S, Churchill R, Aiken L, Bhattacharya A, Gimson A, Brutt AL, de Jong K, Moore M. Routine use of patient reported outcome measures (PROMs) for improving treatment of common mental health disorders in adults. Cochrane Database of Systematic Reviews. 2016 Issue 7. Art No.: CD011119.

[7] Zulfic Z, Liu D, Lloyd C, Rowan J, Schubert KO. Is telepsychiatry care a realistic option for community mental health services during the COVID-19 pandemic? Aust N Z J Psychiatry. 2020 Dec;54(12):1228.

[8] Dunster GP, Swendsen J, Merikangas KR. Real-time mobile monitoring of bipolar disorder: a review of evidence and future directions. Neuropsychopharmacology. 2021 Jan;46(1):197-208.

[9] Wilhelm S, Weingarden H, Ladis I, Braddick V, Shin J, Jacobson NC. Cognitive-Behavioral Therapy in the Digital Age: Presidential Address. Behav Ther. 2020 Jan;51(1):1-14.

[10]Cornet VP, Holden RJ. Systematic review of smartphone-based passive sensing for health and wellbeing. J Biomed Inform. 2018 Jan; 77:120-132.

[11] Asselbergs J, Ruwaard J, Ejdys M, Schrader N, Sijbrandij M, Riper H. Mobile Phone-Based Unobtrusive Ecological Momentary Assessment of Day-to-Day Mood: An Explorative Study. J Med Internet Res. 2016 Mar 29;18(3):e72.

[12] Saeb S, Zhang M, Karr CJ, Schueller SM, Corden ME, Kording KP, Mohr DC. Mobile Phone Sensor Correlates of Depressive Symptom Severity in Daily-Life Behavior: An Exploratory Study. J Med Internet Res. 2015 Jul 15;17(7):e175.

[13]Depp CA, Moore RC, Dev SI, Mausbach BT, Eyler LT, Granholm EL. The temporal course and clinical correlates of subjective impulsivity in bipolar disorder as revealed through ecological momentary assessment. J Affect Disord. 2016 Mar 15;193:145-50.

[14] Granholm E, Loh C, Swendsen J. Feasibility and validity of computerized ecological momentary assessment in schizophrenia. Schizophr Bull. 2008 May;34(3):507-14.

[15] Orlowski S, Lawn S, Matthews B, Venning A, Wyld K, Jones G, Winsall M, Antezana G, Schrader G, Bidargaddi N. The promise and the reality: a mental health workforce perspective on technology-enhanced youth mental health service delivery. BMC Health Serv Res. 2016 Oct 10;16(1):562.

[16] Thompson WK, Gershon A, O'Hara R, Bernert RA, Depp CA. The prediction of study-emergent suicidal ideation in bipolar disorder: a pilot study using ecological momentary assessment data. Bipolar Disord. 2014 Nov;16(7):669-77.

[17]Faurholt-Jepsen M, Vinberg M, Frost M, Debel S, Margrethe Christensen E, Bardram JE, Kessing LV. Behavioral activities collected through smartphones and the association with illness activity in bipolar disorder. Int J Methods Psychiatr Res. 2016 Dec;25(4):309-323.

[18]Faurholt-Jepsen M, Frost M, Vinberg M, Christensen EM, Bardram JE, Kessing LV. Smartphone data as objective measures of bipolar disorder symptoms. Psychiatry Res. 2014 Jun 30;217(1-2):124-7.

[19] Komatsu H, Sekine Y, Okamura N, Kanahara N, Okita K, Matsubara S, Hirata T, Komiyama T, Watanabe H, Minabe Y, Iyo M. Effectiveness of Information Technology Aided Relapse Prevention Programme in Schizophrenia excluding the effect of user adherence: a randomized controlled trial. Schizophr Res. 2013 Oct;150(1):240-4.

[20] Spaniel F, Vohlídka P, Hrdlicka J, Kozený J, Novák T, Motlová L, Cermák J, Bednarík J, Novák D, Höschl C. ITAREPS: information technology aided relapse prevention programme in schizophrenia. Schizophr Res. 2008 Jan;98(1-3):312-7.

[21] aan het Rot M, Hogenelst K, Schoevers RA. Mood disorders in everyday life: a systematic review of experience sampling and ecological momentary assessment studies. Clin Psychol Rev. 2012 Aug;32(6):510-23.

[22]Braun V, Clarke V. Using thematic analysis in psychology. Qual Res Psychol. 2006;3(2):77-101.

[23]Kroenke K, Spitzer RL, Williams JB. The PHQ-9: validity of a brief depression severity measure. J Gen Intern Med. $2001 \mathrm{Sep} ; 16(9): 606-13$. 
[24] Spitzer RL, Kroenke K, Williams JB, Löwe B. A brief measure for assessing generalized anxiety disorder: the GAD-7. Arch Intern Med. 2006 May 22;166(10):1092-7.

[25] Mundt JC, Marks IM, Shear MK, Greist JH. The Work and Social Adjustment Scale: a simple measure of impairment in functioning. Br J Psychiatry. 2002 May;180:461-4.

[26]Pinfold V, Cotney J, Hamilton S, Weeks C, Corker E, Evans-Lacko S, Rose D, Henderson C, Thornicroft G. Improving recruitment to healthcare research studies: clinician judgements explored for opting mental health service users out of the time to change viewpoint survey. J Ment Health. 2019 Feb;28(1):42-48.

[27]Barch DM, Pagliaccio D, Luking K. Mechanisms Underlying Motivational Deficits in Psychopathology: Similarities and Differences in Depression and Schizophrenia. Curr Top Behav Neurosci. 2016;27:41149.

[28] Glasgow RE, Harden SM, Gaglio B, Rabin B, Smith ML, Porter GC, Ory MG, Estabrooks PA. RE-AIM Planning and Evaluation Framework: Adapting to New Science and Practice With a 20-Year Review. Front Public Health. 2019 Mar 29;7:64.

[29]Feijt MA, de Kort YA, Bongers IM, IJsselsteijn WA. Perceived Drivers and Barriers to the Adoption of eMental Health by Psychologists: The Construction of the Levels of Adoption of eMental Health Model. J Med Internet Res. 2018 Apr 24;20(4):e153.

[30] Vis C, Mol M, Kleiboer A, Bührmann L, Finch T, Smit J, Riper H. Improving Implementation of eMental Health for Mood Disorders in Routine Practice: Systematic Review of Barriers and Facilitating Factors. JMIR Ment Health. 2018 Mar 16;5(1):e20.

[31] Musker M, Short C, Licinio J, Wong ML, Bidargaddi N. Using behaviour change theory to inform an innovative digital recruitment strategy in a mental health research setting. J Psychiatr Res. 2020 Jan;120:113. 